\title{
Combination of TPF regimen and cinobufotalin inhibits proliferation and induces apoptosis in human hypopharyngeal and laryngeal squamous cell carcinoma cells
}

This article was published in the following Dove Medical Press journal:

OncoTargets and Therapy

\author{
Xixi Shen' \\ Yong Tao ${ }^{2}$ \\ Yifan Yang' \\ Ru Wang' \\ Jugao Fang' \\ Meng Lian' \\ 'Department of Otorhinolaryngology \\ Head and Neck Surgery, Beijing \\ Tongren Hospital, Capital Medical \\ University, Beijing 100730, China; \\ ${ }^{2}$ Department of Pharmacy, Liaocheng \\ Third People's Hospital, Liaocheng, \\ Shandong 252000, China
}

\begin{abstract}
Background: Hypopharyngeal squamous cell carcinoma and laryngeal squamous cell carcinoma have a potential risk of metastasis and local recurrence. It is urgent to determine novel effective therapy.

Methods: UMSCC5 and FADU cells were treated with TPF regimen (a mixture comprising paclitaxel, cisplatin, and 5-fluorouracil), cinobufotalin, and combination. Cell counting kit-8 (CCK-8), clone formation assays, and flow cytometry were performed to investigate whether combination of cinobufotalin and TPF can exhibit synergistic effects in reducing tumor growth. Results: CCK- 8 assay and $\mathrm{IC}_{50}$ analysis showed that the TPF and cinobufotalin could suppress viability of UMSCC5 and FADU cells, implying the strong antitumor effect. The synergism between TPF and cinobufotalin was further verified by the CCK- 8 and clone formation assays showing the TPF- and cinobufotalin-suppressed cell proliferation synergistically. Notably, flow cytometry showed that the combination also promoted apoptosis synergistically.

Conclusion: TPF regimen combining cinobufotalin suppressed proliferation and promoted apoptosis synergistically in hypopharyngeal squamous cell carcinoma and laryngeal squamous cell carcinoma cells.
\end{abstract}

Keywords: TPF regimen, cinobufotalin, hypopharyngeal and laryngeal squamous cell carcinoma, proliferation, apoptosis

\section{Introduction}

Hypopharyngeal squamous cell carcinoma (HSCC) and laryngeal squamous cell carcinoma (LSCC) are common head and neck SCCs, accounting for $2 \%-6 \%$ of all head and neck cancers. ${ }^{1,2}$ Both HSCC and LSCC are considered life-threatening diseases because of the risk of metastasis to cervical lymph nodes, lack of evident clinical symptoms, and local infiltration during early stages, challenging the treatment of HSCC and LSCC. ${ }^{3}$ Therefore, there is an urgent need to determine novel effective methods to diagnose and treat HSCC and LSCC.

Chemotherapy has been considered a clinical strategy for the treatment of cancer owing to the advantages such as induction of cell death and inhibition of cancer cell migration or metastasis. Paclitaxel is the best natural anticancer drug that has been found to date, and it has been widely used clinically to treat breast, ovarian, partial head and neck, and lung cancers., ${ }^{4,5}$ 5-Fluorouracil, an uracil analog, inhibits cancer by suppressing DNA synthesis. It is widely used as a chemotherapeutic drug for various cancers, including colorectal cancer, ${ }^{6}$ pancreatic cancer, ${ }^{7}$ and hepatocellular carcinoma. ${ }^{8}$
Correspondence: Jugao Fang; Meng Lian Department of Otolaryngology Head and Neck Surgery, Beijing Tongren Hospital, Capital Medical University, Ist Dongjiaominxiang Street, Dongcheng District, Beijing 100730, China Email fangjugaol965III0@163.com; menglian1986I222@I63.com (c)
hereby accept the Terms. Non-commercial uses of the work are permitted without any further permission from Dove Medical Press Limited, provided the work is properly attributed. For permission for commercial use of this work, please see paragraphs 4.2 and 5 of our Terms (https://www.dovepress.com/terms.php). 
Platinum-based agents are used extensively for cancer treatment via DNA adduction. ${ }^{9}$ However, the therapeutic efficacy of these chemotherapeutics is severely restricted by drug resistance, resulting in the failure of chemotherapy. Moreover, chemotherapy is associated with pain and side effects on various organ systems. Bleomycin results in pulmonary damage, anthracyclines cause cardiotoxicity, and platinumbased agents cause nephrotoxic lesions, vomiting, and diarrhea. ${ }^{10}$ To reduce the side effects and improve therapeutic efficiency, regimens comprising a combination of drugs are a better choice because the synergistic effect of different drugs helps to overcome resistance. A combination of 5-fluorouracil and cisplatin (FP regimen) is widely used as a standard treatment for head and neck cancer, and it shows good efficacy. ${ }^{11}$ Although chemotherapy shows favorable efficacy, side effects and drug resistance remain major obstacles in cancer therapy.

Recently, traditional Chinese medicine has received widespread attention owing to its potential application in anticancer therapy. Certain active ingredients of traditional Chinese medicine can boost the immune system and induce cancer cell differentiation and apoptosis with fewer complications and secondary syndromes than chemotherapy drugs. ${ }^{12,13}$ For example, herbal-derived medicines have been used as novel drugs to minimize systemic side effects induced by chemotherapy. ${ }^{4}$ Cinobufotalin, known as cardiotoxic bufanolide steroid, is the main component extracted from Asiatic toad (Bufo gargarizans). Cinobufotalin injection is widely used in the treatment of liver cancer, lung cancer, and other malignant tumors, as well as in the auxiliary treatment of tumor-related complications, with fewer side effects. ${ }^{14,15}$ It has been reported that cinobufotalin inhibits tumor growth and triggers apoptosis by regulating the expression of Bax and Bcl-2 in MCF-7 cells. ${ }^{14}$ In addition, cinobufotalin exerts local analgesic effect by activating the POMC/ $\beta$-END/ $\mu$-OR pathway in cancer tissues. ${ }^{16}$ However, it has not been determined whether cinobufotalin alone or in combination with other drugs can be used for the treatment of head and neck SCC.

In the present study, the effects of TPF regimen, a mixture comprising cisplatin, paclitaxel, and 5-fluorouracil; cinobufotalin; and their combination were investigated. This study may provide a novel perspective on the treatment of HSCC and LSCC and show that a therapeutic regimen comprising or combining traditional Chinese medicine could serve as a novel therapeutic regimen with higher efficacy and fewer side effects.

\section{Materials and methods}

\section{Cell lines and cell culture}

The HSCC cell line FADU was gifted by the Cell Laboratory of Beijing Institute of Otolaryngology, and the LSCC cell line UMSCC5 was gifted by the University of Michigan, Ann Arbor, MI, USA. The use of the cell lines was approved by ethics committee of Beijing Tongren Hospital. The cells were cultured in DMEM (Invitrogen, Carlsbad, CA, USA) supplemented with $10 \% \mathrm{FBS}$ and $1 \%$ penicillin/streptomycin (Invitrogen) $(100 \mathrm{U} / \mathrm{mL})$ in an incubator at $37^{\circ} \mathrm{C}$ with $5 \% \mathrm{CO}_{2}$.

\section{Preparation of TPF solution}

$\mathrm{IC}_{50}$ of cisplatin, paclitaxel, and 5-fluorouracil had been determined in the previous experiments. ${ }^{17}$ The components and preparation of TPF are presented in Table 1. TPF stock solution was diluted $10^{4}$ times when used.

\section{Cell counting kit-8 (CCK-8) assay}

UMSCC5 or FADU cells were seeded onto a 96-well plate at a density of $1 \times 10^{4}$ cells per well. Serial concentrations of TPF or cinobufotalin were added to the culture medium after 24 hours. The concentration gradient of cinobufotalin for both UMSCC5 and FADU cells was 100, 50, 12.5, 6.25, $3.125,1.5625$, and $0 \mu \mathrm{g} / \mathrm{mL}$. The concentration gradient of TPF for UMSCC5 cells was 62.8, 31.4, 15.7, 7.85, 3.925, and $0 \mu \mathrm{g} / \mathrm{mL}$ and that for FADU cells was 46.85, 23.43, 11.71, $5.85,2.93$, and $0 \mu \mathrm{g} / \mathrm{mL}$. The drug treatments were performed for 3 days. Subsequently, $20 \mu \mathrm{L}$ of CCK-8 reagent (Beyotime Biotechnology, Shanghai, China) per well was added, and the plate was incubated for another 1-4 hours. The spectrometric absorbance at $450 \mathrm{~nm}$ was measured using a microplate reader (BioTek, Winooski, VT, USA), and the $\mathrm{IC}_{50}$ value was calculated graphically relative to cell proliferation in the control group.

Table I The components and preparation of TPF

\begin{tabular}{l|l|l}
\hline & FADU & UMSCC5 \\
\hline $\mathrm{IC}_{50}(\mu \mathrm{g} / \mathrm{mL})$ & & \\
$\mathrm{T}$ & 0.125 & 1.17064 \\
$\mathrm{P}$ & 1.017 & 9.89756 \\
$\mathrm{~F}$ & 232.486 & 296.773 \\
The concentration of & & \\
components in TPF $(\mu \mathrm{g} / \mathrm{mL})$ & & \\
$\mathrm{T}$ & 0.0125 & 0.117 \\
$\mathrm{P}$ & 0.339 & 3.3 \\
$\mathrm{~F}$ & 46.49725 & 59.35 \\
The preparation of TPF & & \\
stock solution $(\mathrm{mg}$ or $\mathrm{mL})$ & & \\
$\mathrm{T}$ & 0.125 & 1.17 \\
$\mathrm{P}$ & 3.39 & 33 \\
$\mathrm{~F}$ & 464.97 & 593.5 \\
DMSO & 10 & 10 \\
Concentration of TPF in & & \\
stock solution $(\mathrm{mg} / \mathrm{mL})$ & & 628 \\
TPF & 468.5 & \\
\hline
\end{tabular}

Abbreviations: F, 5-fluorouracil; P, cisplatin; T, paclitaxel. 


\section{Flow cytometry by annexin V/propidium iodide staining}

UMSCC 5 or FADU cells were seeded onto a six-well plate at a density of $5 \times 10^{5}$ cells per well. The cells were then treated with cinobufotalin or TPF at $\mathrm{IC}_{50}$ for 72 hours, followed by staining with fluorescein isothiocyanate (FITC)-conjugated annexin $\mathrm{V}$ and propidium iodide (PI), according to the instructions in the annexin V-FITC Apoptosis Detection Kit (Beyotime Biotechnology, Shanghai, China). After trypsin digestion, the cells were harvested and washed with PBS and resuspended in binding buffer. Subsequently, $10 \mathrm{~mL}$ of PI and $1.1 \mathrm{~mL}$ of annexin V-FITC were added to the cell suspension and mixed for 20 minutes under dark condition. The apoptosis ratio was measured using the LSR II instrument (BD Biosciences). Data analyses were performed using the CELL Quest software.

\section{Clone formation assay}

UMSCC5 or FADU cells were seeded onto a 96-well plate at a density of $1 \times 10^{4}$ cells per well. After 1 day, the cells were treated with cinobufotalin or $\mathrm{TPF}$ at $\mathrm{IC}_{50}$. Coomassie blue staining was performed after 7 days. The cells were washed twice with PBS, fixed in methanol, and stained with Coomassie blue for 5 minutes. The cells were then washed with PBS and air-dried. The number of colonies was determined by imaging with a Multimage ${ }^{\text {TM }}$ Cabinet (Alpha Innotech Corporation, San Leandro, CA, USA) and using the AlphaEase Fc software.

\section{Statistical analyses}

For cell line studies, all data were repeated for at least three independent experiments. The data are presented as mean \pm SD of five separate experiments and were analyzed by Student's $t$-test. Statistical calculation was performed in GraphPad Prism 6 software. $P$-values $<0.05,<0.01$, or $<0.001$ are indicated by $*, * *$, or $* * *$, respectively.

\section{Results}

\section{TPF and cinobufotalin decreased the viability of UMSCC5 and FADU cells synergistically}

From the results of CCK-8 assay, the $\mathrm{IC}_{50}$ of cinobufotalin was 19.49 and $35.78 \mu \mathrm{g} / \mathrm{mL}$ for FADU and UMSCC5 cell lines (Figure 1A and C), respectively, and that of TPF was 8.14 and $22.12 \mu \mathrm{g} / \mathrm{mL}$ for FADU and UMSCC5 cell lines, respectively (Figure $1 \mathrm{~B}$ and $\mathrm{D}$ ), suggesting significant anticancer effect. To investigate whether TPF shows synergistic effects with cinobufotalin in decreasing cell viability, we performed another series of CCK-8 tests. UMSCC5 cells were treated with serial concentrations of cinobufotalin and a single concentration of TPF (Table 1). The results showed that administration of TPF decreased the $\mathrm{IC}_{50}$ of cinobufotalin (Figure 1C). Next, UMSCC5 cells were treated with serial concentrations of TPF and a single concentration of cinobufotalin. As shown in Figure 1D, the administration of cinobufotalin decreased the $\mathrm{IC}_{50}$ of TPF. The experiments performed with FADU cells presented similar results (Figure 1A and B). These findings reveal that TPF and cinobufotalin could decrease the viability of UMSCC5 and FADU cells synergistically.

Following $\mathrm{IC}_{50}$ determination, we investigated whether cell viability suppression activity of TPF and cinobufotalin is time-dependent. UMSCC5 cells were treated with cinobufotalin, TPF, and both cinobufotalin and $\mathrm{TPF}$ at $\mathrm{IC}_{50}$, or both cinobufotalin and $\mathrm{TPF}$ at half $\mathrm{IC}_{50}$. The control group did not receive drug treatment. The CCK-8 assay was performed, and the $\mathrm{OD}_{450}$ values were measured after 1, 2, and 3 days, respectively. The highest OD value was observed in the control group, suggesting that cell proliferation occurred in a time-dependent manner. When treated with $\mathrm{TPF}$ or cinobufotalin at $\mathrm{IC}_{50}$, the increase in $\mathrm{OD}$ value was negligible. There was almost no increase in the OD value during the 3-day treatment with the combination of TPF and cinobufotalin at $\mathrm{IC}_{50}$. Notably, after 2 days of treatment with a combination of TPF and cinobufotalin at half $\mathrm{IC}_{50}$, the OD value was lower than that of either treatment alone, implying synergistic effects of TPF and cinobufotalin. The experiment with FADU cells demonstrated similar results (Figure 2). These findings indicate that TPF and cinobufotalin suppressed the viability of UMSCC5 and FADU cells synergistically and time-dependently.

To determine synergism of TPF regimen and cinobufotalin in vitro, the Chou-Talalay combination index (CI) values were calculated using the formula reported by $\mathrm{Chou}^{18}$ :

$$
\begin{aligned}
\mathrm{CI} & =\frac{\left(D_{1}\right)}{\left(D_{\mathrm{x}}\right)_{1}}+\frac{(D)_{2}}{\left(D_{\mathrm{x}}\right)_{2}} \\
& =\frac{(D)_{1,2}[P / P+Q]}{\left(D_{\mathrm{m}}\right)_{1}\left[f_{\mathrm{a}} /\left(1-f_{\mathrm{a}}\right)\right]^{1 / m_{1}}}+\frac{(D)_{1,2}[Q / P+Q]}{\left(D_{\mathrm{m}}\right)_{2}\left[f_{\mathrm{a}} /\left(1-f_{\mathrm{a}}\right)\right]^{1 / m_{2}}}
\end{aligned}
$$

where $\left(D_{\mathrm{x}}\right)_{1}$ and $\left(D_{\mathrm{x}}\right)_{2}$ in the denominators are the doses of each drug "alone", which gives $\mathrm{x} \%$ effect (eg, $\mathrm{x} \%$ inhibition); $(D)_{1}$ and $(D)_{2}$ in combination. When cell growth was inhibited $50 \%,(D)_{1}=(D) \mathrm{TPF}=2.69631 \mu \mathrm{g} / \mathrm{mL},\left(D_{\mathrm{x}}\right)_{1}=\left(D_{50}\right)$ $\mathrm{TPF}=8.14333 \mu \mathrm{g} / \mathrm{mL},(D)_{2}=(D)$ cinobufotalin $=3.08145$ $\mu \mathrm{g} / \mathrm{mL},\left(D_{\mathrm{x}}\right)_{2}=\left(D_{50}\right)$ cinobufotalin $=19.4857 \mu \mathrm{g} / \mathrm{mL}$, and 

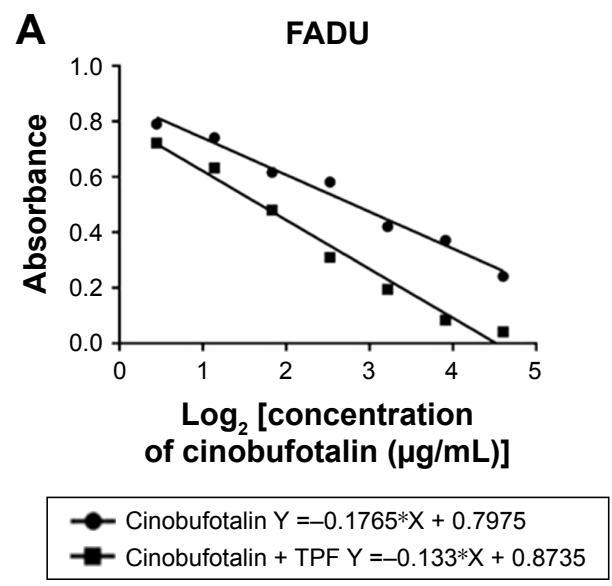

C

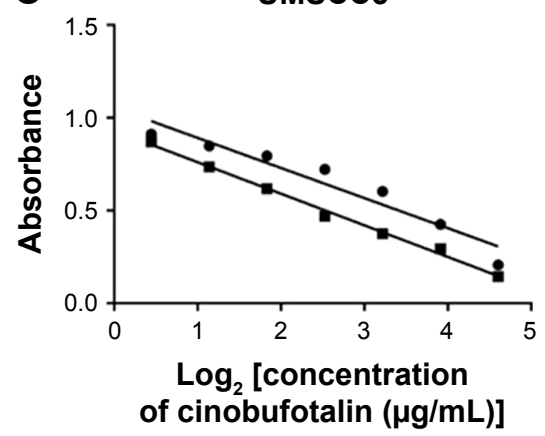

Cinobufotalin $Y=-0.1703 * X+0.9313$

Cinobufotalin + TPF Y= $-0.1623 * X+1.054$
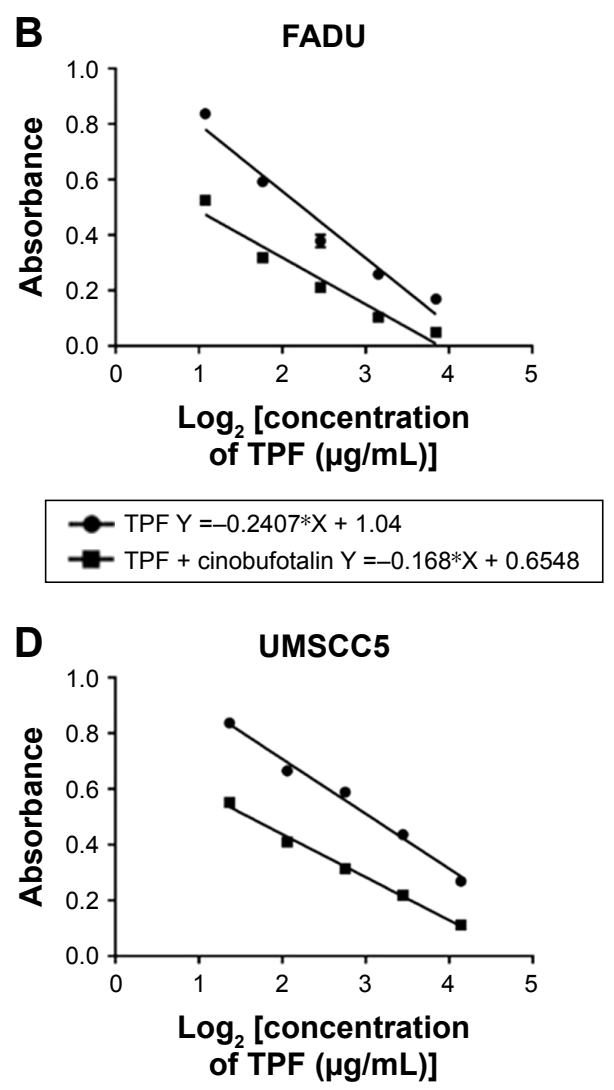

TPF $Y=-0.1969 * X+1.102$

TPF + cinobufotalin $Y=-0.1544 * X+0.7466$

Figure I TPF and cinobufotalin decrease viability of UMSCC5 and FADU cells synergistically.

Notes: (A) FADU cells treated by serial concentrations of cinobufotalin, or combined with $\mathrm{IC}_{50}$ concentration of TPF for 3 days and (B) FADU cells treated by serial concentrations of TPF, or combined with $\mathrm{IC}_{50}$ concentration of cinobufotalin for 3 days were detected by CCK-8 kit for the viability alterations, and the IC $\mathrm{C}_{50}$ is calculated by the viability curve. (C) UMSCC5 cells treated by serial concentrations of cinobufotalin, or combined with IC ${ }_{50}$ concentration of TPF for 3 days and (D) UMSCC5 cells treated by concentrations of TPF, or combined with $\mathrm{IC}_{50}$ concentration of cinobufotalin for 3 days were detected by CCK-8 kit for the viability alterations, and the IC $\mathrm{F}_{50}$ is calculated by the viability curve.

Abbreviations: CCK-8, cell counting kit-8; TPF, a mixture comprising paclitaxel, cisplatin, and 5-fluorouracil.
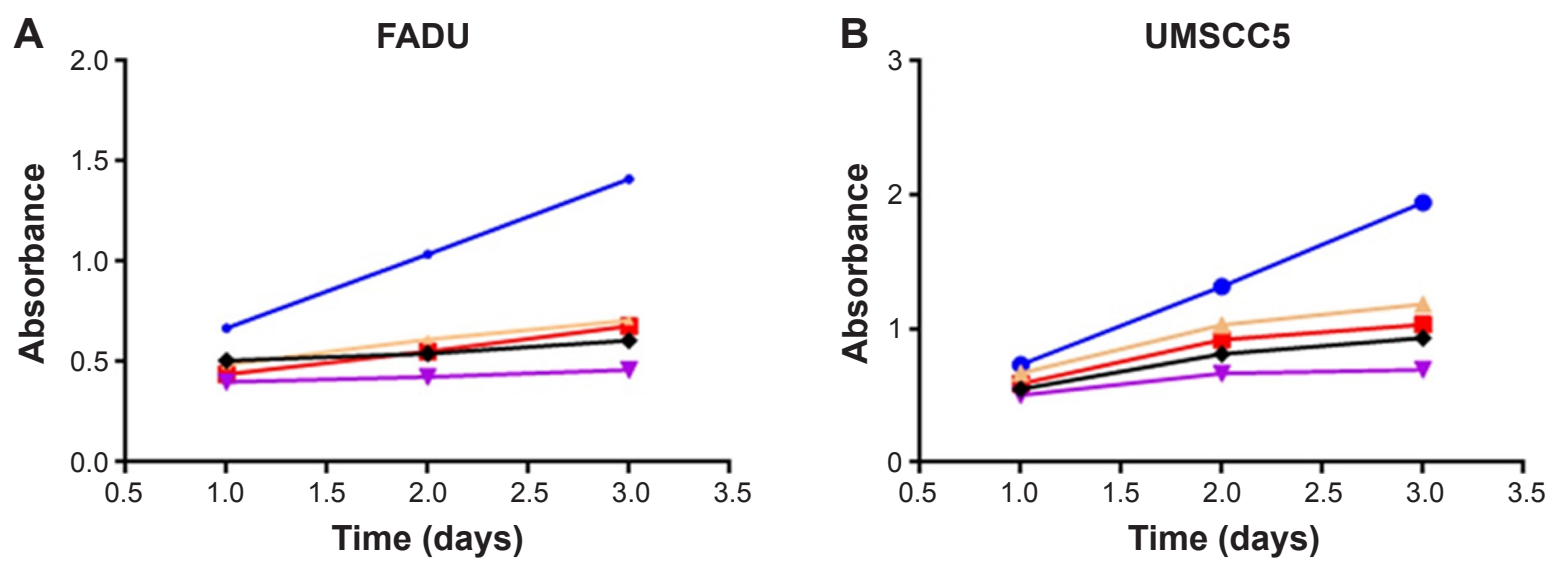

Control $\rightarrow$ TPF $\rightarrow$ Cinobufotalin $\rightarrow$ TPF + cinobufotalin $\rightarrow 1 / 2$ TPF $+1 / 2$ cinobufotalin

Figure 2 TPF and cinobufotalin suppress the viability of UMSCC5 and FADU cells time-dependently.

Notes: (A) FADU and (B) UMSCC5 cells were treated by IC $_{50}$ concentrations of TPF alone, IC $_{50}$ concentrations of cinobufotalin alone, IC ${ }_{50}$ concentration of cinobufotalin with $I C_{50}$ concentration of TPF, or half IC $\mathrm{C}_{50}$ concentration of cinobufotalin with half IC $\mathrm{F}_{50}$ concentration of TPF for I, 2, and 3 days, then were detected by CCK-8 kit for the viability alterations, with no medicine treatment as control, and proliferation curves were drawn according to the variation of the absorbance.

Abbreviations: CCK-8, cell counting kit-8; TPF, a mixture comprising paclitaxel, cisplatin, and 5-fluorouracil. 
the $\mathrm{CI}=0.49<1$, which indicated an synergism effect. Dose reduction index (DRI) was also calculated. For TPF, DRI = $\left(D_{\mathrm{x}}\right)_{1} /(D)_{1}=3.02$. For cinobufotalin, DRI $=\left(D_{\mathrm{x}}\right)_{2} /(D)_{2}=6.32$.

\section{TPF and cinobufotalin synergistically suppressed colony formation of UMSCC5 and FADU cells}

The results of the CCK- 8 assay showed that both cinobufotalin and TPF suppressed cell viability, indicating their synergistic effects in UMSCC5 and FADU cells. To verify whether the inhibition of viability by cinobufotalin and TPF is associated with proliferation, we conducted colony-forming experiments in UMSCC5 and FADU cells. UMSCC5 and FADU cells were treated with cinobufotalin, TPF, or both cinobufotalin and TPF at $\mathrm{IC}_{50}$. The control group did not receive any drug treatment. We observed that cell growth was drastically inhibited when treated with cinobufotalin or TPF, compared to that of the control. The maximum cell growth inhibition was observed when treated with the combination of cinobufotalin and TPF, with only a small number of monoclonal cell clusters remaining (Figure 3 ). These findings suggest that cinobufotalin and TPF suppressed proliferation and that they can synergistically promote antiproliferation effect in UMSCC5 and FADU cells.

\section{TPF and cinobufotalin promote the apoptosis of UMSCC5 and FADU cells synergistically}

Molecular biology has revealed that the imbalance in cell proliferation and apoptosis is an important mechanism of tumorigenesis and progression. ${ }^{19}$ Therefore, apoptosis and proliferation are the two main targets for tumor treatment. We determined the effects of cinobufotalin and TPF on the apoptosis of HSCC and LSCC cells by annexin V-FITC/PI staining. After treatment with cinobufotalin, TPF, or combination of cinobufotalin and TPF at $\mathrm{IC}_{50}$ for 72 hours, FADU cells were analyzed by flow cytometry with annexin $\mathrm{V}$ and PI double staining. The total apoptosis rate was calculated
A
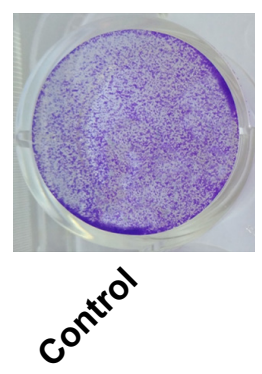

FADU

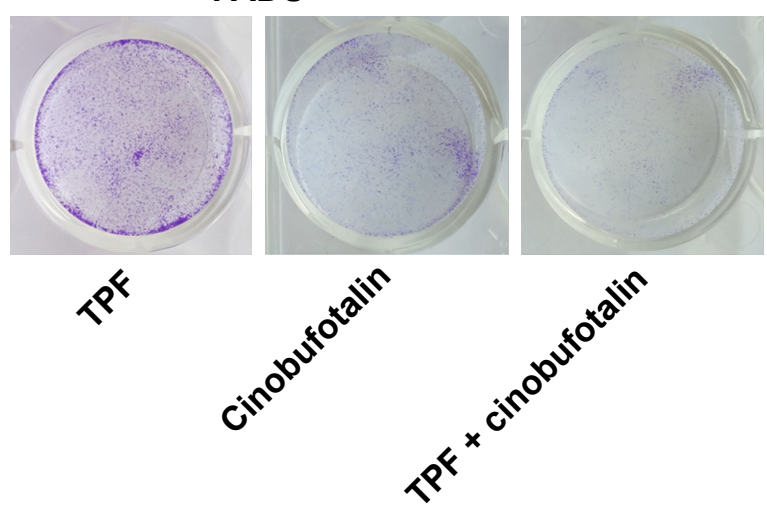

B
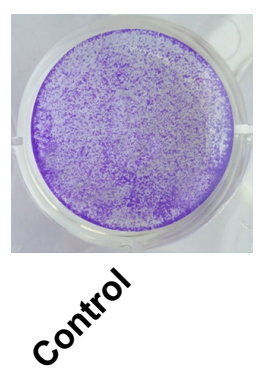

UMSCC5

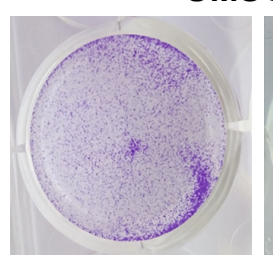

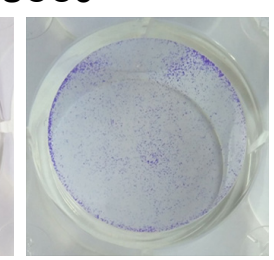

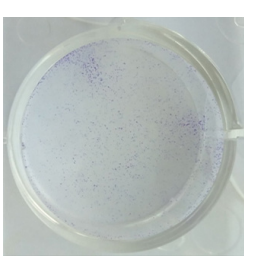

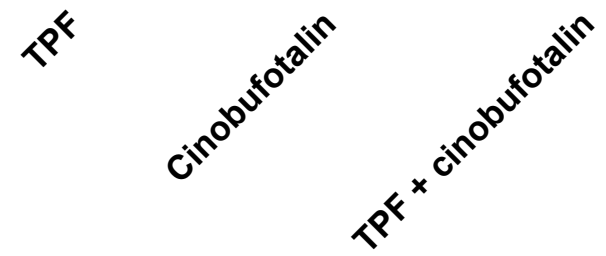
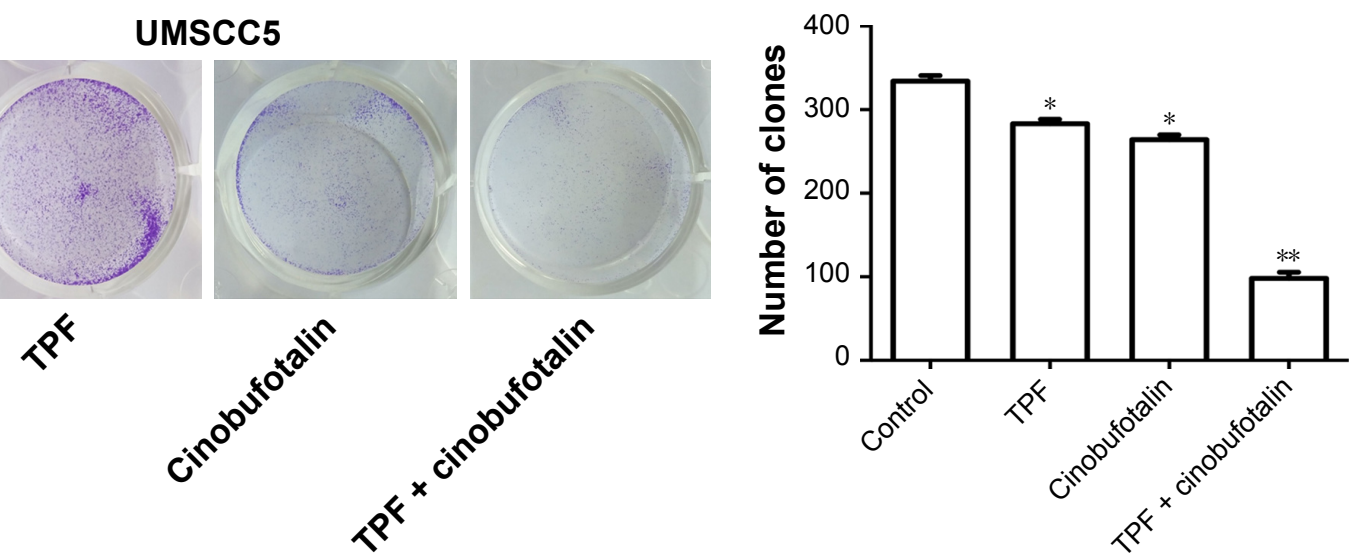

Figure 3 TPF and cinobufotalin suppress colony forming of UMSCC5 and FADU cells.

Notes: (A) FADU and (B) UMSCC5 cells treated with IC $\mathrm{C}_{50}$ concentrations of TPF alone, $\mathrm{IC}_{50}$ concentrations of cinobufotalin alone, combination of IC $\mathrm{C}_{50}$ concentration of cinobufotalin and $\mathrm{IC}_{50}$ concentration of TPF for 7 days were stained by Coomassie blue for the proliferation alterations, with no medicine treatment as control. Representative experiments are shown on the left side and statistical analyses were shown on the right side. The average values \pm SD of three separate experiments were plotted, and significant differences from the control value are indicated by $* P<0.05$ and $* * P<0.01$.

Abbreviation: TPF, a mixture comprising paclitaxel, cisplatin, and 5 -fluorouracil. 
as the percent of early and late apoptotic cells. The percentage of apoptosis after cinobufotalin and TPF treatment alone increased by 9.66 and 8.67 , respectively, suggesting that both cinobufotalin and TPF could promote apoptosis considerably in FADU cells. Combination treatment with cinobufotalin and $\mathrm{TPF}$ at $\mathrm{IC}_{50}$ showed $16.22 \%$ increase in apoptosis ratio. To confirm the proapoptotic role of cinobufotalin and TPF, we conducted the same experiments in UMSCC5 cells and obtained similar results (Figure 4). These findings showed that cinobufotalin and TPF not only inhibited proliferation, but also promoted apoptosis, thus playing an important role in regulating the development of HSCC and LSCC.

\section{Discussion}

In this study, we aimed to determine the effects of combination treatment with TPF regimen and cinobufotalin on HSCC and LSCC cells. We found that the combination treatment inhibited proliferation and induced apoptosis in UMSCC5 and FADU cells.

HSCC and LSCC are life-threatening diseases that affect a patient's breathing and appetite, resulting in increased risk of metastasis and recurrence. Currently, the most effective treatment in clinical settings is surgical extraction followed by chemotherapy. The pharmacokinetics and pharmacokinetic toxicity of chemotherapy drugs in patients with tumor significantly vary with individual. ${ }^{20}$ For example, 5-fluorouracil is a folic acid metabolism inhibitor and has been used as a first-line chemotherapy drug for colon, stomach, and esophageal cancers. Marcuello ${ }^{21}$ showed that single-nucleotide polymorphism in the $5^{\prime}$ tandem repeat sequences of thymidylate synthase gene results in different responses to fluorouracil-based chemotherapy in advanced colorectal cancer. The poor efficiency of chemotherapy drugs is due to the heterogeneity and polymorphism of tumor, and the administration of single chemotherapy drugs cannot exert a considerable effect on tumor development, which brings out the significance of drug combination for specific cancers. Suman Mukhopadhyay mentioned the significance of using 5-FU and paclitaxel along with other drugs in RAS mutated cancer. ${ }^{22}$ The TPF regimen, which combines the advantages of 5-fluorouracil, paclitaxel, and cisplatin, exhibits prominent antitumor effects by inhibiting proliferation and promoting apoptosis, and thereby improving the efficiency of treatment of HSCC and LSCC by overcoming tumor heterogeneity.
A

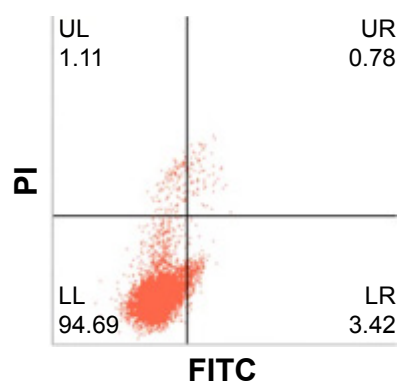

B

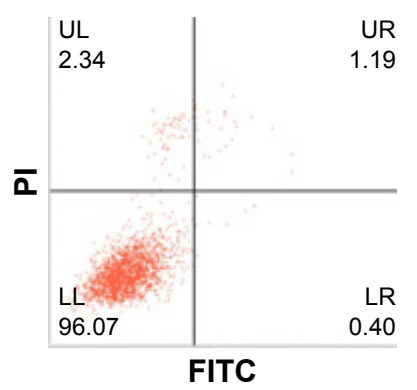

TPF

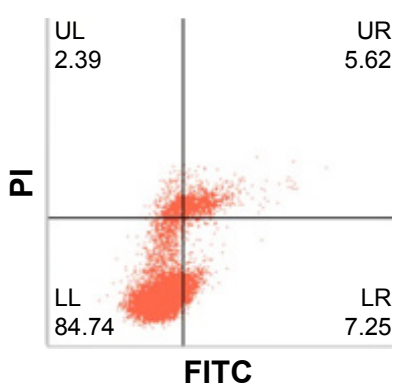

TPF

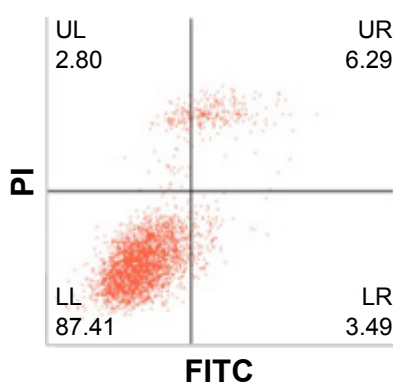

Cinobufotalin

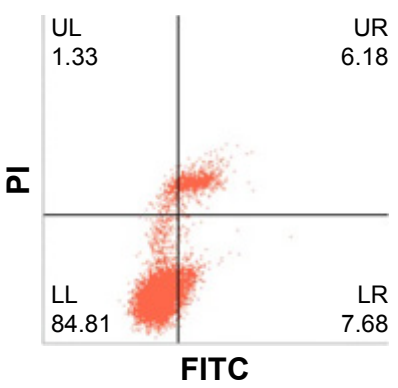

Cinobufotalin

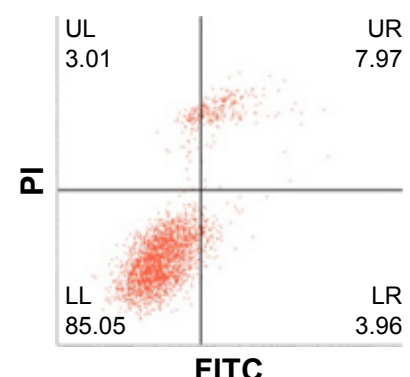

TPF + cinobufotalin

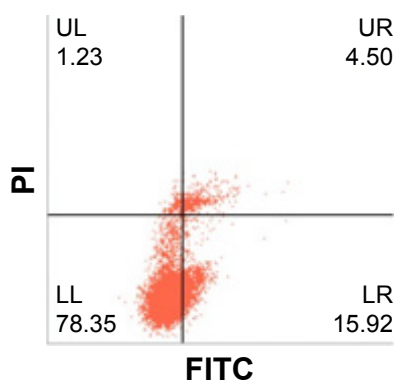

TPF + cinobufotalin

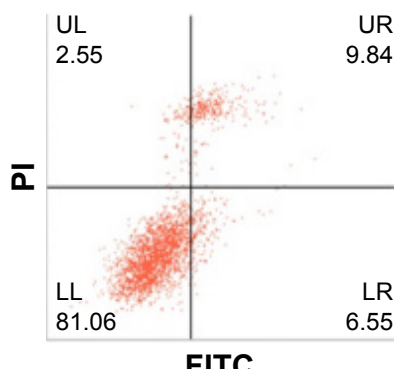

Figure 4 TPF and cinobufotalin promote apoptosis of UMSCC5 and FADU cells.

Notes: (A) FADU and (B) UMSCC5 cells were treated by IC ${ }_{50}$ concentrations of TPF alone, IC $\mathrm{C}_{50}$ concentrations of cinobufotalin alone, combination of IC $\mathrm{C}_{50}$ concentration of cinobufotalin and IC $\mathrm{C}_{50}$ concentration of TPF for 3 days, then were stained with annexin V-FITC with no medicine treatment as control. LL quadrant indicates vital cells (negative for both FITC-annexin and PI). UL quadrant indicates damaged cells (annexin V-negative and PI-positive). UR quadrant indicates slate apoptotic cells (positive for both FITC-annexin V and PI); LR quadrant indicates early apoptotic cells (annexin V-positive and PI-negative).

Abbreviations: FITC, fluorescein isothiocyanate; LL, lower left; LR, lower right; PI, propidium iodide; TPF, a mixture comprising paclitaxel, cisplatin, and 5-fluorouracil; UL, upper left; UR, upper right. 
Although chemotherapy has a certain beneficial effect on malignant tumors, it results in greater toxicity by killing normal cells. In addition, the emergence of drug resistance reduces the efficacy of chemotherapy and a significant number of patients are forced to discontinue treatment. For example, drug resistance leads to high recurrence and poor five-year survival rate in patients with ovarian cancer. ${ }^{23}$ Although radiotherapy is used for the local treatment of tumor, its efficacy in different malignant tumors is different. Chinese traditional medicine can be used for the treatment of malignant tumors. It can inhibit cancer cell growth with little damage to normal cells, improve patient health, stimulate the body's immune system, and reduce toxic side effects of chemotherapy and radiotherapy, thereby significantly improving the quality of life of patients with cancer. Cinobufotalin has been reported to inhibit proliferation and induce apoptosis in U2OS cells via the ROS/JNK/ p38 signaling pathway. ${ }^{24}$ Yuan reported that cinobufotalin significantly enhanced the sensitivity of p-gp substrate drugs to p-gp-overexpressing cells but had no effect on the parental cells. Cinobufotalin enhanced the effect of doxorubicin (DOX) against p-gp-overexpressing LoVo/ADR cell xenografts in nude mice. In addition, cinobufotalin increased the apoptotic effects of chemotherapy agents, as well as the intracellular accumulation of DOX and Rho123 in MDR cells. These findings suggest that cinobufotalin can be further developed into a safe and potent modulator for combination use with anticancer drugs in cancer chemotherapy. ${ }^{15}$ However, the role of cinobufotalin in HSCC and LSCC has not yet been determined. To the best of our knowledge, the present study revealed the anticancer effect of cinobufotalin in HSCC and LSCC for the first time. Cinobufotalin suppressed the proliferation and promoted the apoptosis of UMSCC 5 and FADU cells. It also increased TPF regimen-induced inhibition of proliferation and promotion of apoptosis of both UMSCC5 and FADU cells. These findings suggest that cinobufotalin is a promising candidate drug and can be considered a safe and potent adjuvant for combination chemotherapy for HSCC and LSCC.

Our in vitro experiments had some limitations. First, for a preclinical study, in vivo validation may provide sufficient proof for applicability. Second, the underlying mechanisms of synergistic action of TPF regimen and cinobufotalin remain elusive. Although we conducted cytobiologic experiments, a deeper understanding of the biology of cinobufotalin and the signaling pathways involved in cell proliferation and apoptosis is needed.

\section{Conclusion}

In summary, our findings showed that the TPF regimen exerts potent antitumor effects via the inhibition of proliferation and promotion of apoptosis of HSCC and LSCC cells. TPF could overcome tumor heterogeneity by combining the advantages of 5-fluorouracil, paclitaxel, and cisplatin. In addition, to the best of our knowledge, we report for the first time that cinobufotalin suppressed proliferation and promoted apoptosis of UMSCC 5 and FADU cells. Subsequent experiments indicated that cinobufotalin can increase TPF regimen-induced inhibition of proliferation as well as promotion of apoptosis of both UMSCC5 and FADU cells, thereby providing novel insights into effective therapy for HSCC and LSCC.

\section{Abbreviations}

TPF, a mixture comprising paclitaxel, cisplatin, and 5-fluorouracil; HSCC, hypopharyngeal squamous cell carcinoma; LSCC, laryngeal squamous cell carcinoma; PI, propidium iodide; DOX, doxorubicin.

\section{Acknowledgment}

Sponsored by Beijing Municipal Administration of Hospitals' Ascent Plan (DFL20180202), Beijing Municipal Administration of Hospitals Incubating Program (PX2018009), Beijing Administration of Traditional Chinese Medicine (QN2018-32).

\section{Disclosure}

The authors report no conflicts of interest in this work.

\section{References}

1. Ligier K, Belot A, Launoy G, et al. Descriptive epidemiology of upper aerodigestive tract cancers in France: incidence over 1980-2005 and projection to 2010. Oral Oncol. 2011;47(4):302-307.

2. Takes RP, Strojan P, Silver CE, et al. Current trends in initial management of hypopharyngeal cancer: the declining use of open surgery. Head Neck. 2012;34(2):270-281.

3. Zafereo M. Surgical salvage of recurrent cancer of the head and neck. Curr Oncol Rep. 2014;16(5):386.

4. Chavoshi H, Vahedian V, Saghaei S, Pirouzpanah MB, Raeisi M, Samadi $\mathrm{N}$. Adjuvant therapy with silibinin improves the efficacy of paclitaxel and cisplatin in MCF-7 breast cancer cells. Asian Pac J Cancer Prev. 2017;18(8):2243-2247.

5. Wanebo HJ, Lee J, Burtness BA, et al. Induction cetuximab, paclitaxel, and carboplatin followed by chemoradiation with cetuximab, paclitaxel, and carboplatin for stage III/IV head and neck squamous cancer: a phase II ECOG-ACRIN trial (E2303). Ann Oncol. 2014;25(10):2036-2041.

6. Wang K, Liu L, Zhang T, et al. Oxaliplatin-incorporated micelles eliminate both cancer stem-like and bulk cell populations in colorectal cancer Int J Nanomedicine. 2011;6:3207-3218.

7. Li X, Ma T, Zhang Q, et al. Modified-FOLFIRINOX in metastatic pancreatic cancer: a prospective study in Chinese population. Cancer Lett. 2017;406:22-26.

8. Hashimoto A, Tanaka T, Sho M, et al. Adjuvant hepatic arterial infusion chemotherapy after resection for pancreatic cancer using coaxial catheter-port system compared with conventional system. Cardiovasc Intervent Radiol. 2016;39(6):831-839.

9. Kim S, Jary M, André T, et al. Docetaxel, cisplatin, and 5-fluorouracil (DCF) chemotherapy in the treatment of metastatic or unresectable locally recurrent anal squamous cell carcinoma: a phase II study of French interdisciplinary GERCOR and FFCD groups (Epitopes-HPV02 study). BMC Cancer. 2017;17(1):574. 
10. Gudaitytė J, Dvylys D, Šimeliūnaite I. Anaesthetic challenges in cancer patients: current therapies and pain management. Acta Med Litu. 2017;24(2):121-127.

11. Ahn D, Sohn JH, Kim JH, Lee JE, Park SH, Kim JC. Concurrent chemoradiotherapy with capecitabine/cisplatin versus 5-fluorouracil/ cisplatin in resectable laryngohypopharyngeal squamous cell carcinoma. Ear Nose Throat J. 2016;95(2):E34-E42.

12. Zhu P, Wu Y, Yang A, Fu X, Mao M, Liu Z. Catalpol suppressed proliferation, growth and invasion of CT26 colon cancer by inhibiting inflammation and tumor angiogenesis. Biomed Pharmacother. 2017;95: $68-76$.

13. Schmidt M, Polednik C, Roller J, Hagen R. Cytotoxicity of herbal extracts used for treatment of prostatic disease on head and neck carcinoma cell lines and non-malignant primary mucosal cells. Oncol Rep. 2013;29(2):628-636.

14. Zhu L, Chen Y, Wei C, et al. Anti-proliferative and pro-apoptotic effects of cinobufagin on human breast cancer MCF-7 cells and its molecular mechanism. Nat Prod Res. 2018;32(4):493-497.

15. Yuan Z, Shi X, Qiu Y, et al. Reversal of P-gp-mediated multidrug resistance in colon cancer by cinobufagin. Oncol Rep. 2017;37(3): 1815-1825.

16. Chen T, Yuan S, Wan XN, et al. Chinese herb cinobufagin-reduced cancer pain is associated with increased peripheral opioids by invaded CD3/4/8 lymphocytes. Oncotarget. 2017;8(7):11425-11441.
17. Jie Z, Ru W, Haizhou W. Effect of c-FOS for induction chemosensitivity in hypopharyngeal squamous cell carcinoma. Chin Arch Otolaryngol Head Neck Surg. 2018;25(4):199-202.

18. Chou TC. The mass-action law based algorithms for quantitative econogreen bio-research. Integr Biol. 2011;3(5):548-559.

19. Lee JH, Hanaoka M, Kitaguchi Y, et al. Imbalance of apoptosis and cell proliferation contributes to the development and persistence of emphysema. Lung. 2012;190(1):69-82.

20. Elsayed YA, Sausville EA. Selected novel anticancer treatments targeting cell signaling proteins. Oncologist. 2001;6(6):517-537.

21. Marcuello E, Altés A, del Rio E, César A, Menoyo A, Baiget M. Single nucleotide polymorphism in the $5^{\prime}$ tandem repeat sequences of thymidylate synthase gene predicts for response to fluorouracil-based chemotherapy in advanced colorectal cancer patients. Int J Cancer. 2004; 112(5):733-737.

22. Mukhopadhyay S, Saqcena M, Foster DA, Suman M, Mahesh S, David AF. Synthetic lethality in KRas-driven cancer cells created by glutamine deprivation. Oncoscience. 2015;2(10):807-808.

23. Li T, Li D, Sha J, Sun P, Huang Y. MicroRNA-21 directly targets MARCKS and promotes apoptosis resistance and invasion in prostate cancer cells. Biochem Biophys Res Commun. 2009;383(3):280-285.

24. Qi F, Inagaki Y, Gao B, et al. Bufalin and cinobufagin induce apoptosis of human hepatocellular carcinoma cells via Fas- and mitochondriamediated pathways. Cancer Sci. 2011;102(5):951-958.
OncoTargets and Therapy

\section{Publish your work in this journal}

OncoTargets and Therapy is an international, peer-reviewed, open access journal focusing on the pathological basis of all cancers, potential targets for therapy and treatment protocols employed to improve the management of cancer patients. The journal also focuses on the impact of management programs and new therapeutic agents and protocols on

\section{Dovepress}

patient perspectives such as quality of life, adherence and satisfaction The manuscript management system is completely online and includes a very quick and fair peer-review system, which is all easy to use. Visit http://www.dovepress.com/testimonials.php to read real quotes from published authors. 